\title{
Superresolution with the Focused Plenoptic Camera
}

\author{
Todor Georgiev ${ }^{b}$, Georgi $\mathrm{Chunev}^{a}$, and Andrew Lumsdaine ${ }^{a}$ \\ ${ }^{a}$ Indiana University, Bloomington, IN, USA; \\ ${ }^{b}$ Adobe Systems, San Jose, CA, USA
}

\begin{abstract}
Digital images from a CCD or CMOS sensor with a color filter array must undergo a demosaicing process to combine the separate color samples into a single color image. This interpolation process can interfere with the subsequent superresolution process. Plenoptic superresolution, which relies on precise sub-pixel sampling across captured microimages, is particularly sensitive to such resampling of the raw data. In this paper we present an approach for superresolving plenoptic images that takes place at the time of demosaicing the raw color image data. Our approach exploits the interleaving provided by typical color filter arrays (e.g., Bayer filter) to further refine plenoptic sub-pixel sampling. Our rendering algorithm treats the color channels in a plenoptic image separately, which improves final superresolution by a factor of two. With appropriate plenoptic capture we show the theoretical possibility for rendering final images at full sensor resolution.
\end{abstract}

Keywords: Focused plenoptic camera, full sensor resolution, super-resolution, Bayer array

\section{INTRODUCTION}

Because of the rich data it captures, the plenoptic camera offers intriguing possibilities for photography. ${ }^{1,2}$ The principles behind the plenoptic camera are not new - the basic ideas were developed by Lippman in $1908^{3,4}$ ). However, with the recent advent of very large-scale image sensors and ubiquitous processing power (particularly via GPUs), the potential of plenoptic cameras can now be practically realized.

Since a plenoptic camera samples the entire 4D radiance of a scene, pixels must be allocated in both spatial and angular dimensions. ${ }^{5}$ With the hand-held plenoptic camera and basic rendering algorithms, the resolution of pictures rendered from a plenoptic image was fixed at the number of microlenses in the camera. ${ }^{6,7}$ Plenoptic cameras garnered an unfortunate reputation for being low resolution as a result. The focused plenoptic camera and corresponding rendering algorithms were recently introduced, allowing pictures to be rendered with multiple pixels per microlens. ${ }^{8}$ However, since the focused plenoptic camera relies on relatively large microlenses, it still produces rendered images that are about a factor of 10 smaller than the sensor resolution.

Since plenoptic cameras capture multiple views of the same scene, superresolution would appear to be an attractive approach to obtaining yet higher resolution. The first results applying superresolution to the traditional and focused plenoptic camera were reported in ${ }^{9}$ and, ${ }^{10}$ respectively.

There are many different methods for applying super resolution in general, but the unifying principle is to use multiple lower resolution images of a given scene to produce a single higher resolution image. ${ }^{11,12}$ If the lower resolution images are shifted by sub-pixel amounts relative to each other, a higher resolution image can be obtained via appropriate registration and combination of the lower-resolution images. This process is then typically followed by deconvolution to compensate for the blur of pixel sampling in the lower resolution images (as well as other imperfections in the optical system).

In this paper we develop a novel super-resolution algorithm that uses raw color image data (i.e., data that has not been demosaiced). Our approach exploits the fact that digital images from a CCD or CMOS sensor with a color filter array are actually acquired as an interlaced set of four separate images. In normal processing, these images undergo a demosaicing process that combines them into a single color image. Our approach instead applies superresolution to the multiple microimages, treating each color channel separately, which provides two important benefits. First, this approach avoids the interpolation that occurs in typical demosaicing, allowing superresolution to be applied to lower resolution images that are sampled sparsely. Second, the resulting color planes are perfectly registered with each other, enabling much more straightforward creation of final color images. By applying superresolution in conjunction with demosaicing in this way, we are able to improve resolution by a factor of two relative to previous superresolution methods. 


\subsection{Related Work}

Superresolution has a rich literature - see, e.g. ${ }^{11,12}$ for surveys. The specific application of superresolution to plenoptic cameras was presented in ${ }^{9}$ and. ${ }^{10}$

The approach presented in ${ }^{9}$ applies superresolution to the sub-sampled images obtained at each of the different views captured across the microlens array of a traditional plenoptic camera. The authors develop a sophisticated image formation and restoration model and apply a blind deconvolution restoration approach. The approach in ${ }^{10}$ is based specifically on the focused plenoptic camera which uses a different image formation model than for the traditional plenoptic camera. Based on this model, a camera design is presented that is guaranteed to produce sub-pixel offsets between microlens images for objects at optical infinity. Neither of these approaches takes into account the fine sampling available in the raw image data.

Superresolution and demosaicing have been combined in the traditional imaging arena. ${ }^{13-16}$ The approaches use the properties of color filter array sampling to improve superresolution while at the same time improving demosaicing. The approach we describe in this paper is similar to these, but applied in the realm of plenoptic cameras. Moreover, because of the precise alignment of the microimages in the focused plenoptic camera, we are able to obtain extremely well-registered images to work with, a feature not available when superresolution is applied to "macro" images.

\section{PLENOPTIC CAPTURE}

Traditionally the plenoptic function is captured with an array of cameras, each taking a picture of the scene from a slightly different perspective. ${ }^{2,17}$ The focused plenoptic camera takes the same approach, only it is based on an array of microcameras implemented with microlenses close to the sensor. ${ }^{8}$

As discussed in, ${ }^{18}$ the focused plenoptic camera is a relay system, where the microlenses re-image pieces of the main lens image multiple times onto the sensor. Due to the very precise geometry of both the microlens array and the sensor pixels, they map parts of the main lens image onto the sensor in an orderly way, and with great precision. Our goal in this paper is to use the exact subpixel alignment of those microimages to extract resolution far greater than what has been captured directly with the sensor in each microimage. ${ }^{9}$ Our first step is to analyze the plenoptic capture model in the case of array of microcameras with a common sensor.

A schematic of the focused plenoptic camera is shown in Figure 1. The main camera lens creates its image at a plane which we call "object space". Capturing the radiance in object space is easily achieved with an array of identical microcameras. Each camera is focused on the object space and captures a different overlapping piece of the object space.

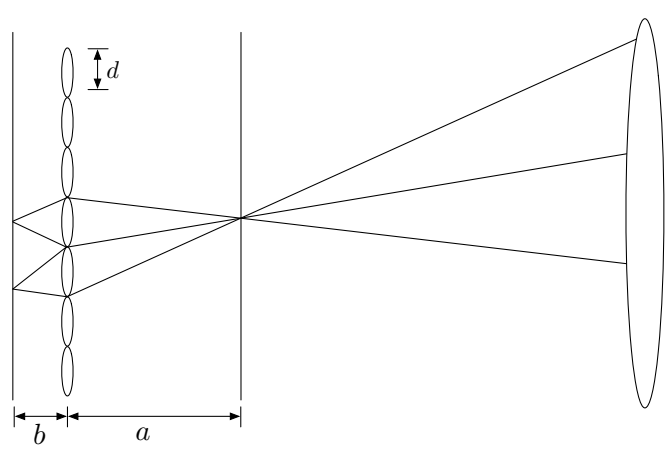

Figure 1. The microlens array works as an array of cameras capturing multiple subimages from the image plane of the main camera lens.

An optical phase space diagram for the radiance in object space, as captured with one single microcamera, is shown Figure 2. The size of the image created in the microcamera is $d$. This size is constrained by the main lens aperture, which needs to be chosen appropriately by matching the $F$-number of the main lens system to that of the microlenses. ${ }^{7}$ The distances $a$ and $b$ define the minification of the microcamera. Considering this fact, the 
object space size of the image for our microcamera is $d \times \frac{a}{b}$. The viewing angle of the microcamera is that size of the object divided by the distance to the object $a$, which can be shown to equal $\frac{d}{b}$. Each point is viewed by the microcamera from a range of angles depending on the microlens aperture. That range of angles is $\frac{d}{a}$.

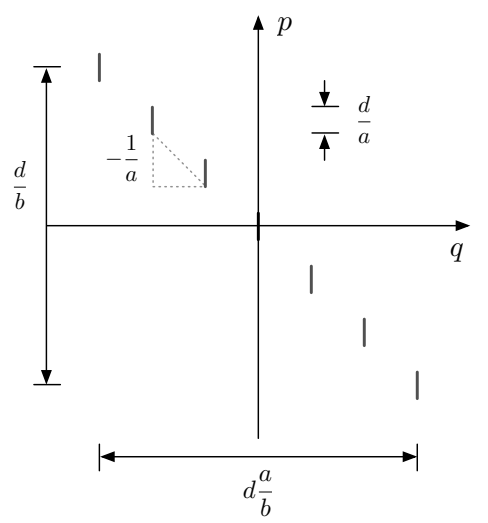

Figure 2. Phase space diagram of the radiance captured by a single microcamera.

Note that different points in object space are seen by the camera at different angles. This is represented by the tilt of the line of pixels depicting the microimage in Figure 2.

If we consider an array of microcameras, the phase space diagram of the radiance captured from object space is represented in Figure 3. An array of cameras that captures radiance in this fashion was first proposed by Lippmann in $1908^{4}$ in relation to his ideas of integral photographs. We will call it "the Lippmann sensor".

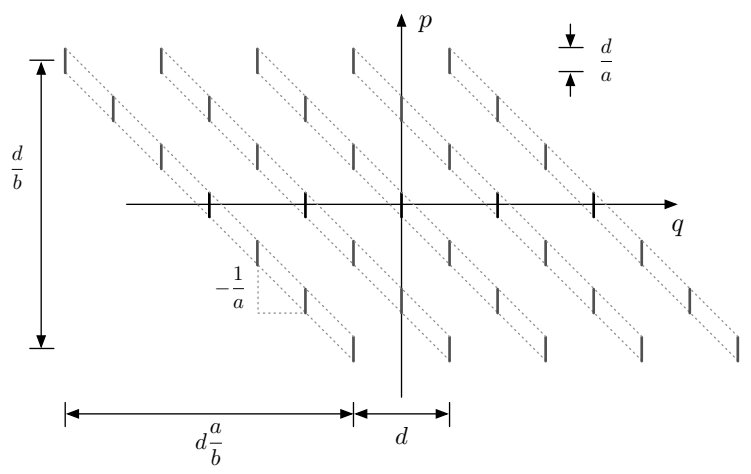

Figure 3. Phase space diagram of the array of microcameras.

This diagram describes the sampling of the radiance by a Lippmann sensor. Notice that pixels are assumed to have infinitely small size in space, which is in line with traditional sampling theory. ${ }^{19}$ However their angular size, which depends on the microlens aperture, is $\frac{d}{a}$.

In the following sections we characterize the maximal spatial resolution that is achievable with a Lippmann sensor using superresolution, including the effect on resolution of finite pixels.

\section{PLENOPTIC SAMPLING}

Plenoptic sampling will be analyzed in the spatial domain considering the two main processes that take place. First, light rays illuminate a given pixel of finite size. The pixel is characterized by its response function to both position and angle for each ray. It produces a total output signal proportional to the sum of the responses to all rays. Second, we are digitally sampling the multiple arrays of pixels under all microlenses, using appropriate kernels based on our interpretation of the capturing process. 


\subsection{Pixel Response to Light}

A pixel responds differently to the same light rays if they hit different locations on it. This response function is nonzero and relatively flat within much of the central area of the pixel and falls off to zero outside the pixel. For instance, CMOS pixels respond substantially only within a fraction of the pixel area, due to the special use of the rest of the real estate for amplifiers and other electronics with purposes other than light sensing. Often a microlens is placed on top of the pixel in order to improve its fill factor, which defines essentially what fraction of the pixel area is responding to light.

In general, the above can be abstracted as a response function of $q$ and $p$, which is the same for every pixel, but specific to the type of sensor used. To simplify derivations we will be assuming that the response function is constant within the range of angles at which our pixels work (the $F$-number of microlenses). Typically, this range is 20 degrees for pixels with microlenses and 40 degrees for CCD pixels without microlenses.

\subsection{Digital Sampling of Pixels}

According to basic sampling theory ${ }^{19}$ pixels are assumed to be of point size, i.e. delta functions of $q$. Perfect sampling is achieved by convolution with a sinc function of appropriate size, $S(x, y)$, or some approximation of the sinc-such as bicubic. This is motivated by the fact that in frequency representation the Fourier transform of the sinc function cuts off high frequencies and we achieve antialiasing.

In plenoptic rendering, pixels that are assembled together in the final image belong to multiple microimages. Considering Figure 5 (Left), during capture we optically sample the object image plane with different microcameras to generate different microimages. Then rendering needs to do its best to reproduce the original image, starting from those captured microimages. Assuming the object image is in focus, this is a perfect setting for superresolution.

Image rendering is a process opposite to image capture. When we put pixels together in a final image we need to consider that they are so dense that their initial response functions overlap. The rendering process corresponding to Figure 5 (Left) is shown in Figure 7 (Left). Note that just as the images of pixels in object space overlap (Figure 5), they are mixed together in rendering (Figure 7). In this sense the output signal of a plenoptic camera is equal to the true optical signal convolved with the pixel response function as a kernel, $K(x, y)$, and taken at the fixed locations of pixels.

Because of that, we need to apply the inverse $K^{-1}$ of the kernel $K$ to the pixel values we have read with our code. In more detail, sampling needs to be done by a kernel that is of the form $S * K^{-1}$, the convolution of the traditional sinc sampling function with the inverse of $K$. In general, the result looks like a sharpening kernel.

\section{PLENOPTIC SUPERRESOLUTION}

If we are imaging a point in $3 D$ with the Lippmann sensor, we would observe multiple copies of that point in the final image. See Figure 1. Those copies appear at certain pitch from each other depending on the distance (depth) to the point in $3 D$. For example depth of infinity results in parallel projections in all microcameras and pitch exactly equal to the distance $d$ between microlenses. A point at closer distance $a$ would result in slightly greater pitch because rays "fan out" and are no longer parallel. In other words, depth is related to pitch in the microimages.

On the other hand, looking at Figure 3 it is clear that different pitch would result in different tilt of the line of projection. For example, depth of infinity would be related to exactly vertical projection onto the $q$ axis with pitch equal to the microlens pitch. Closer distance would be related to greater pitch and correspondingly slanted lines of projection.

The basic idea in our superresolution approach can be explained in relation to Figure 4, which for simplicity assumes point pixels (both in position and angle). Choosing the right angle of projection can produce density of pixels in the final rendered image much higher than the density in the original microimages.

In our approach we implement this projection, or construction of the final image, in terms of sampling. Consider how the Lippmann sensor takes a snapshot of a planar image created in front of it by the main lens. Figure 5 shows two diagrams of the portion of such an image plane sampled by the pixels of neighboring 


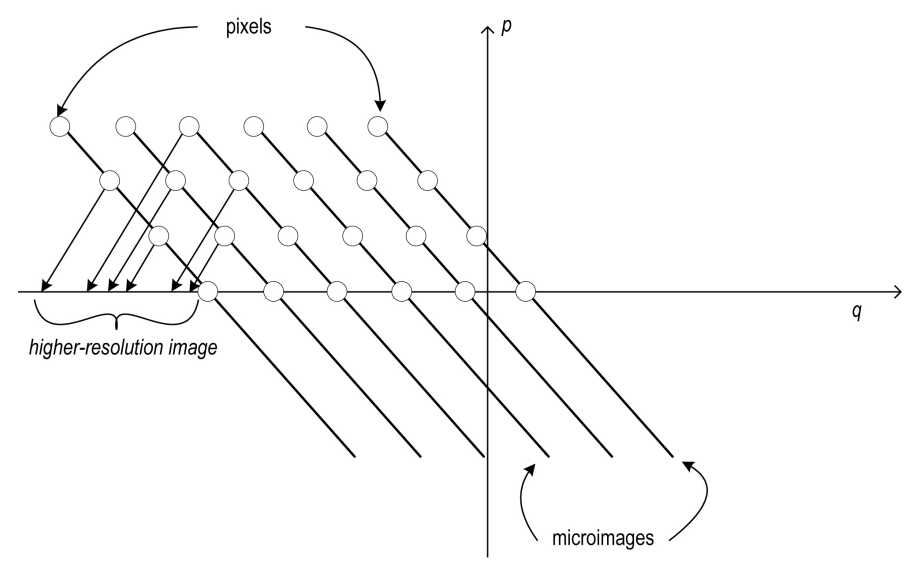

Figure 4. Projections can produce images with higher resolution than that of the original microimages.

microimages. Figure 5 (left) depicts the case of large fill factor pixels. The inverse of the depicted capture process can be used to construct an output image focused at the given image plane, and, if there is some sub-pixel offset between the captured data in neighboring microimages, the resulting output image can be super-resolved.

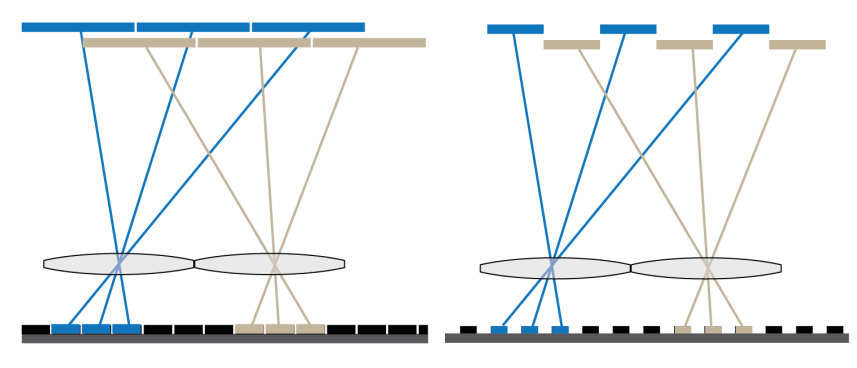

Figure 5. Left: Shows the sampling of one image plane by the pixels of two neighboring microimages. Right: Same as the left subfigure, but with pixels that have fill factor 0.5 .

This is done by sampling from the pixels from all microimages with a given kernel along a given line of projection. See Figure 6. In more detail, we choose the pitch (focusing). Then we produce the output pixel by normalized blending of all pixels from all microimages with the kernel weights according to the distance to each pixel. The problem is greatly simplified by the fact that the kernel is zero beyond certain small radius. Also at a reasonable pitch we get no more than 50 microimages that intersect with the line of projection.

Another depiction of the rendering process for the captured data can be seen from the phase space diagrams, figure 7 , corresponding to figure 5 . Note that, when using full fill rate pixels to produce final super-resolved images we still need to deconvolve the results. As shown in figures 5 and 7 right, reducing the fill factor of the pixels on the sensor can remove the need for deconvolving the output. With this type of approach, full sensor resolution can be achieved for a given image plane. All that needs to be done is use small enough pixels and interleave all microimages that capture a focused view of the object plane. The main point is that input pixels for that given plane do not overlap at the output.

Our results from grayscale tests are shown in figure 9. We have captured a 39 megapixel plenoptic image with a grayscale sensor. A crop from the captured grayscale image is shown in Figure 8 (left). It is a picture of a boat behind a volleyball net. Notice that all microimages are inverted because microcameras captures inverted images.

We have split the image into groups of 3X3 pixels. From every such group we have taken only the central pixel. This simulates a sensor with small pixels with spaces between pixels. A crop from this image can be seen 

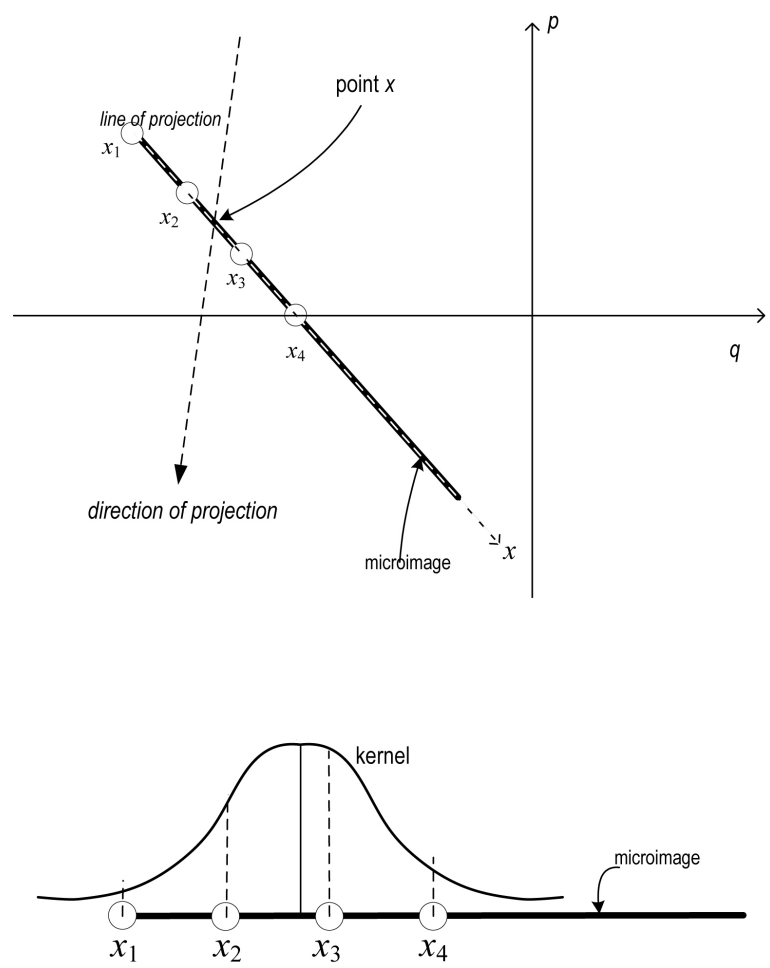

Figure 6. Top: shows the line of sampling relative to the pixels in one microimage. Bottom: Sampling kernel is centered at point $\mathrm{x}$, where the output pixel is generated.
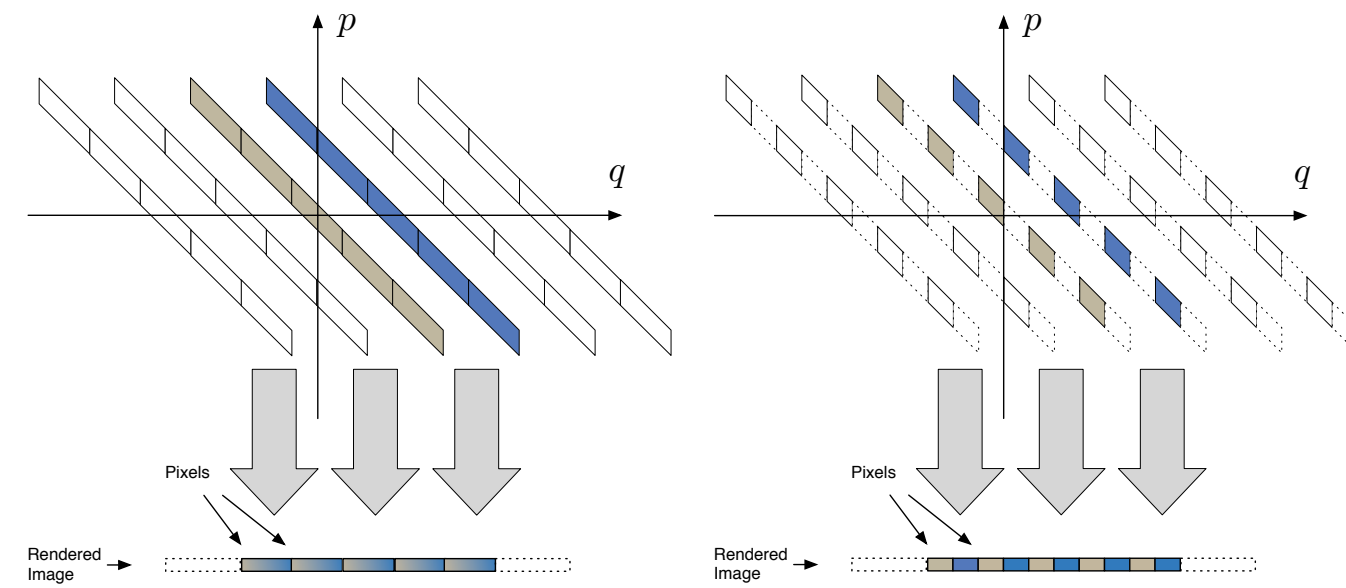

Figure 7. Phase space diagrams corresponding to the two radiance capture processes depicted in figure 5. The superresolved image synthesized from the full fill rate pixels shown in the left subfigure requires deconvolution, while the image rendered from the reduced fill rate pixels shown in the right subfigure is inherently sharp.

in Figure 8 (right). Note that the empty space between pixels is not represented because we are showing how an image actually captured with such a sensor would look like. Note also that we have lost any obvious evidence of the net. This makes our superresolution result even more amazing: Not only do we see the net and the boat clearly, but we also freely refocus on them with very little artifacts.

Figure 10 depicts a simplified version of the superresolution rendering process. Pixels from different microimages are interleaved into the final superresolved image without any need for deconvolution. It is clear that this method is general, and not limited to just blocks of 3X3. With sufficiently small pixels we can interleave a very 

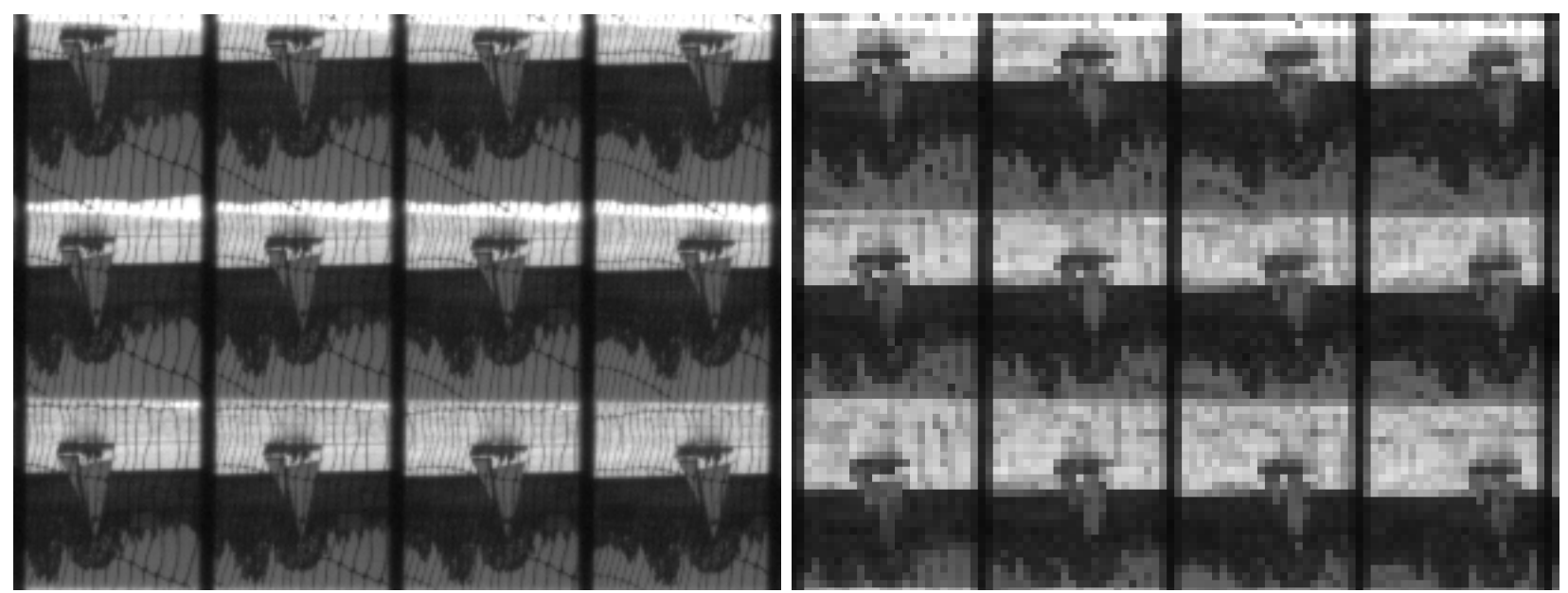

Figure 8. Left: a crop from an intact gray scale plenoptic image. Right: Taking only the central pixel from every group of $3 \times 3$ pixels from the crop shown in the left subfigure. This simulates image captured by a sensor with small pixels and gaps between them. This image was used for the direct and superresolved rendering shown below.
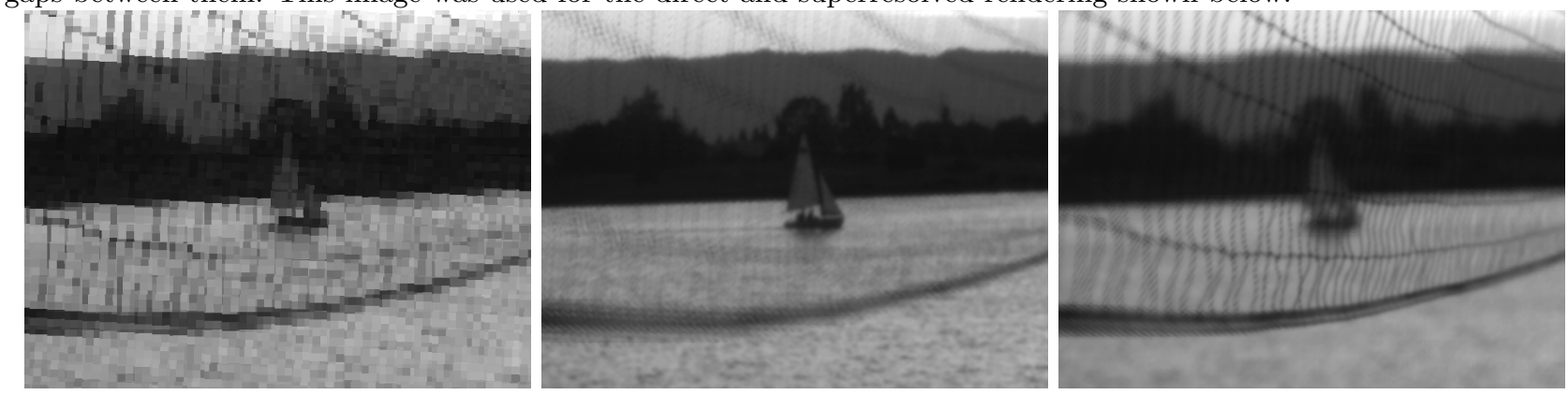

Figure 9. Results from superresolving (fill factor 1/9). In order to simulate low fill factor pixels we are using only the central pixel out of every group of $3 x 3$ pixels in a grayscale sensor. Left: Direct nearest neighbor sampling where each pixel comes from a single microimage. Middle: Same image as on the left, only superresolved with a pitch focused on the boat. Right: Same image as on the left, only superresolved with a pitch focused on the net.

large number of microimages and even reach full sensor resolution when rendering the plane that's in focus. The obvious problem is that such sensors are not available.

For this paper we develop a practical use of the above technique in the case of superresolved demosaicing of plenoptic images captured with a standard color sensor with a Bayer color filter array .

\section{DEMOSAICING}

In the case of color images, a typical workflow has been to perform demosaicing first, at the time of opening the image, and only then other operations. It turns out that in the case of plenoptic superresolution this approach produces suboptimal results. That's because an effective subpixel shift is typically introduced in all demosaicing algorithms. Instead of being performed in two discrete steps, demosaicing and super-resolving need to be performed together, which prevents the loss of information resulting from other processing pipelines.

A simplified model of the basics of typical demosaicing is represented in Figure 11. We see that for each color channel pixels need to be inserted in places where such pixels are missing. If this is done in a generic way without regard for the specifics of plenoptic capture, the involved effective kernel interferes with superresolution.

Directly superresolving undemosaiced focused plenoptic data produces higher resolution than superresolving after the desmosaicing for two reasons. First, plenoptic sampling can be applied to each color component, giving high weights to the closest captured values for a given color component; and second the pixels that capture a given color component can be interleaved with little overlap, because of the skipping that occurs due to the 

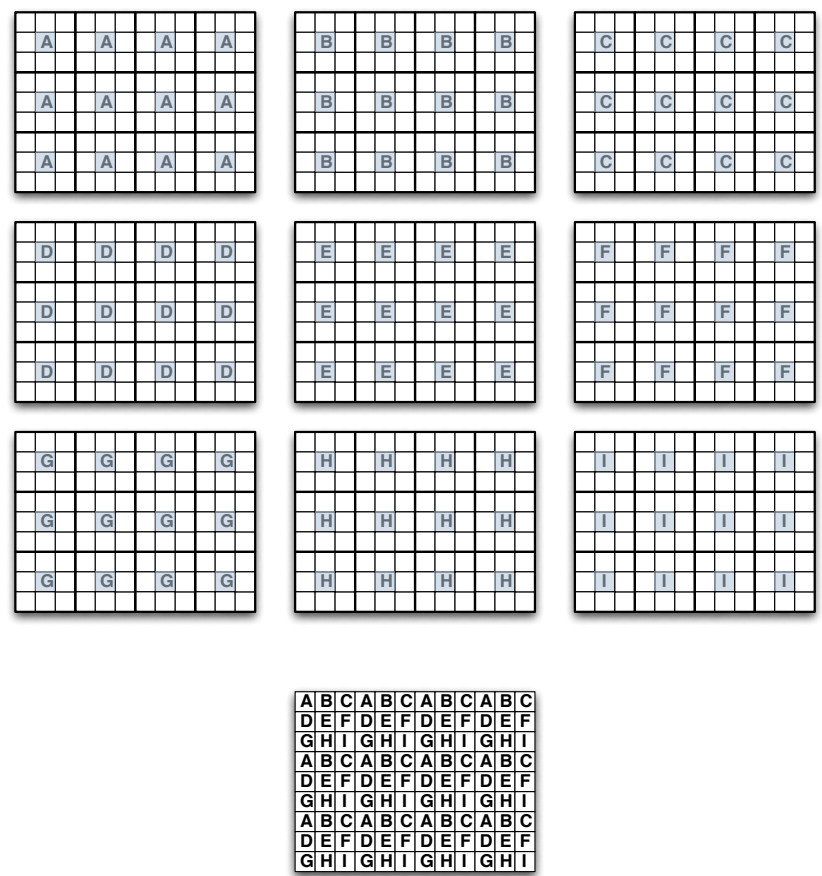

Figure 10. Top: Conceptually shows 9 microimages, where 8 pixels out of every group of 9 pixels are not used (depicted in white). The pixel that is used is denoted with a letter. Bottom: Superresolved rendering into the final image is done by just copying, without any deconvolution.

color filter array. As was demonstrated in the previous sections, plenoptic sampling produces the superresolution effect, while having space between pixels removes some of the need for deconvolution, resulting in very fast superresolution algorithms. For a depiction of the demosaicing process see Figures 11 and 12.

Our demosaicing results are shown in the Experimental Results section. We chose to take only the nearest sample for a given color component from each microimage and blend it with the nearest samples from other microimages, with dependance on the distance to each pixel according to a Gaussian kernel. The results can be improved by sampling a larger number of pixels from each microimage and by adapting standard demosaicing techniques, like detecting edges to avoid blending overlapping objects. Such experiments go beyond the scope of this paper and could be of interest for future research on the topic.

\section{CAMERA AND RENDERING}

Our camera is a medium format camera with a 39-megapixel digital back from Phase One. We are using two sensors: Color and grayscale. They are two versions of KAF 39000 from Kodak: with and without Bayer array filters. The $80-\mathrm{mm}$ lens is mounted on the camera with a $13-\mathrm{mm}$ extension tube, which provides the required spacing $a$ between the microlens array and the main lens image.

The microlens array is custom made by Leister Axetris, with focal length $1.5 \mathrm{~mm}$, pitch of $500 \mu \mathrm{m}$, and precision $1 \mu \mathrm{m}$. The sensor pixels are $6.8 \mu \mathrm{m}$. The value of $b \approx 1.6 \mathrm{~mm}$ was estimated with precision $0.1 \mathrm{~mm}$ from known sensor parameters and independently from the microlens images at different $\mathrm{F} /$ numbers.

The microlens apertures are formed by a black chromium mask deposited on the microlenses and are circular, each with a diameter of $100 \mu \mathrm{m}$. While this small aperture size extends the depth of field and makes the microlenses diffraction limited, it also introduces a high $\mathrm{F} /$ number, with associated diffraction blur and longer required exposure times. Note that these small apertures are not required in general, especially with microlenses of good optical quality. The pitch of the microlenses is chosen in order to match the F-number of the main camera lens, which is $F / 3$ and defined by a hand-made square aperture that we inserted at the location of the original lens aperture. 


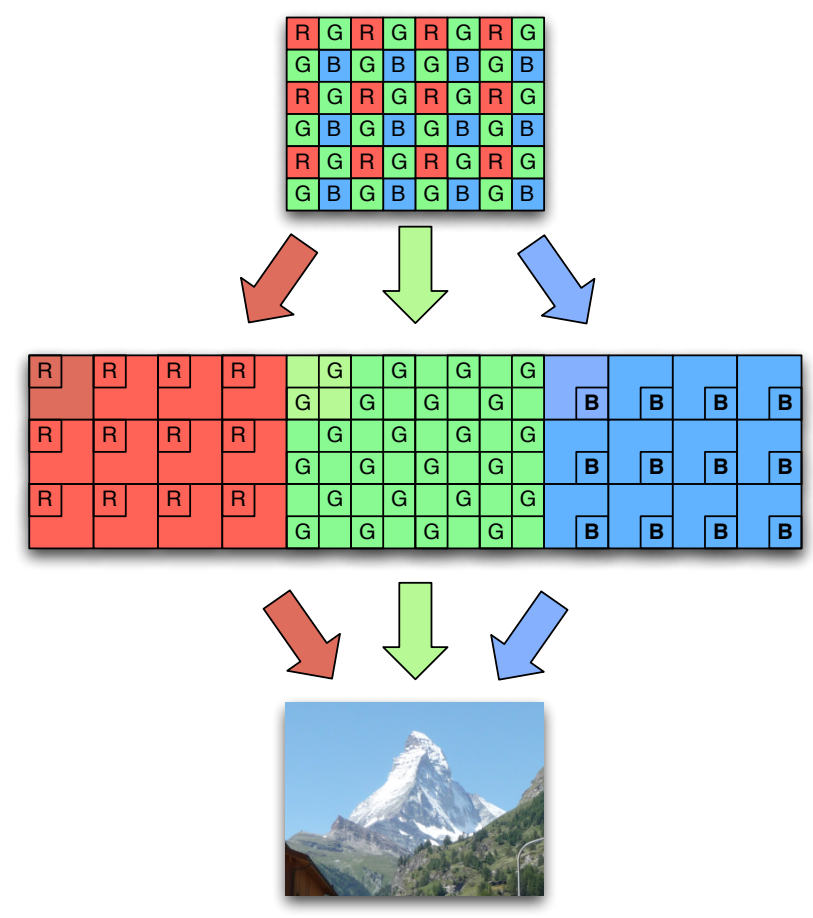

Figure 11. Conceptually illustrates basic Bayer array demosaicing.

We have implemented our algorithms using OpenGL and the GL shader language to take advantage of GPU performance. We are rendering the final image with a modified version of the blending algorithm described ${ }^{18}$ so that we take into account the Bayer filter array. Appropriate sampling and blending with a Gaussian kernel is implemented as discussed in the previous sections. Best results in terms of sharp superresolution are achieved with kernel width equal to 0.1 of the pixel size, or smaller. The number of microimages sampled for generating each output pixel is typically on the order of 50. Depending on the number of microimages sampled, we achieve full screen image rendering at approximately 2 to 5 frames per second on an average desktop machine.

\section{EXPERIMENTAL RESULTS}

We have taken a color picture of a tree and a car behind it with our 39 megapixel sensor that uses a standard Bayer color filter array. Figure 13 (left) shows a crop containing some of the microimages for the license plate of the car. This is the image as opened in Photoshop, with a standard demosaicing algorithm. Figure 13 (right) shows a crop from the corresponding raw image file. Bayer colors are provided only for visual representation that marks the red, green, and blue channels.

Figure 14 (left) shows superresolution with a traditional Bayer array color processing. In the middle, we see results from the same picture, but using the raw data for demosaicing while superresolving. The image on the right demonstrates that we can still perform refocusing without strong artifacts. In other words, superresolution does not prevent us from achieving standard lightfield effects.

In another experiment we worked with the raw data from the superresolution camera of Georgiev and Lumsdaine, ${ }^{10}$ as shown in Figure 15 (data used with permission). Figure 16 shows the rendering results. With this experiment we can also see a significant improvement in resolution. Notice in particular the swings in the background. In the right figure, the structure of the chains holding the swings can be made out. In the left figure, and as shown in, ${ }^{10}$ that structure is not visible. In the input data (the individual microimages) the structure of the chains is also not detectable visually. 


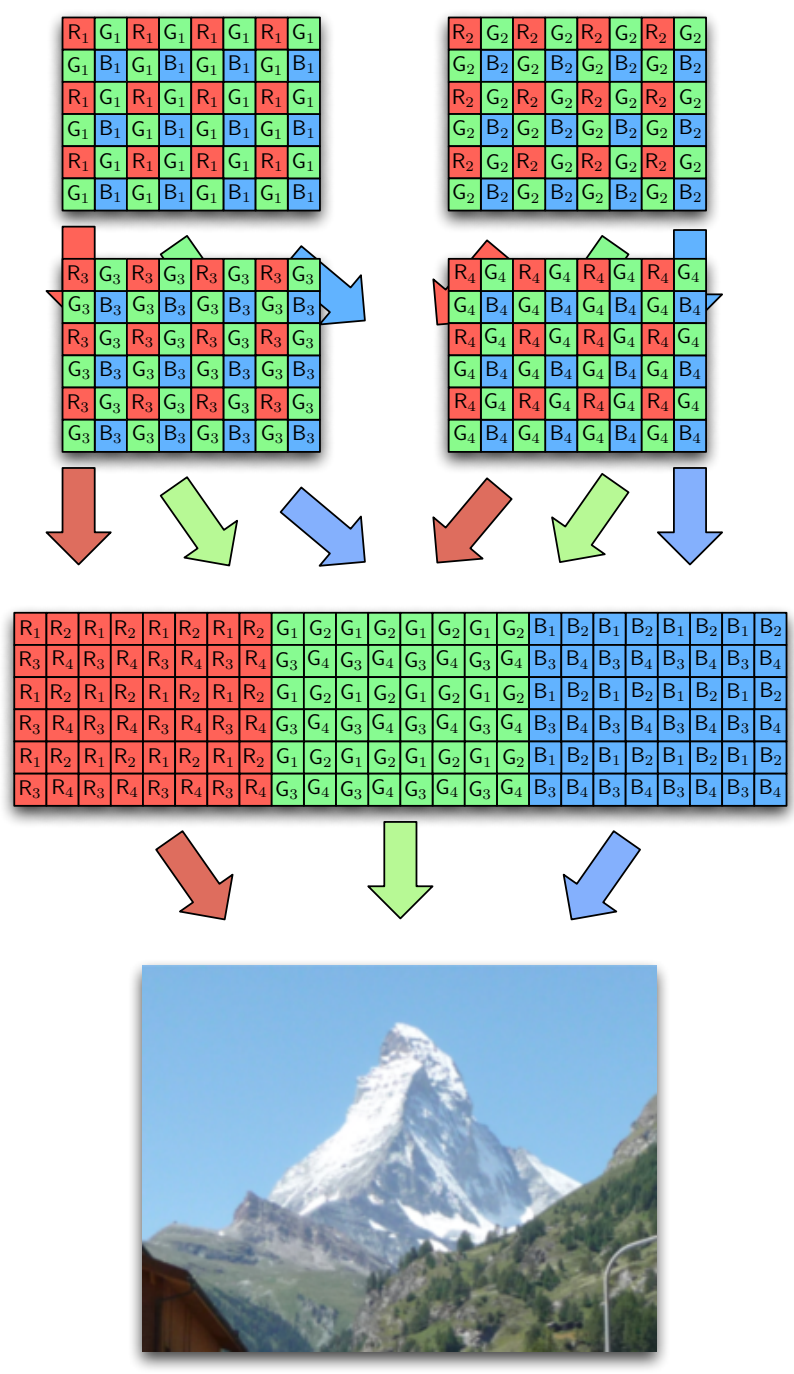

Figure 12. Illustrates basic plenoptic demosaicing. The resolution of each color channel is at least doubled, resulting in a higher final image resolution.

\section{CONCLUSION}

The radiance (or plenoptic function) carries a significant amount of information. Although plenoptic cameras have received significant attention because of the novel effects that can be obtained when rendering, the information captured by these cameras can also be used to increase the spatial resolution of rendered images — while still allowing the novel rendering effects.

The focused plenoptic camera makes deliberate spatio-angular trade-offs and provides reasonable spatial resolution. With the application of super-resolution techniques as presented in this paper, we are able to push the attainable spatial resolution even further. In the case of color images we improve final resolution by a factor of two, and as a theoretical result we show possibility of full sensor resolution rendering with appropriately captured plenoptic data. One factor that has limited the adoption of plenoptic cameras until now has been the relatively low available resolution. The combination of the focused plenoptic camera with superresolution enables images of sizes acceptable to modern photographers, making lightfield photography significantly more practical and appealing. 

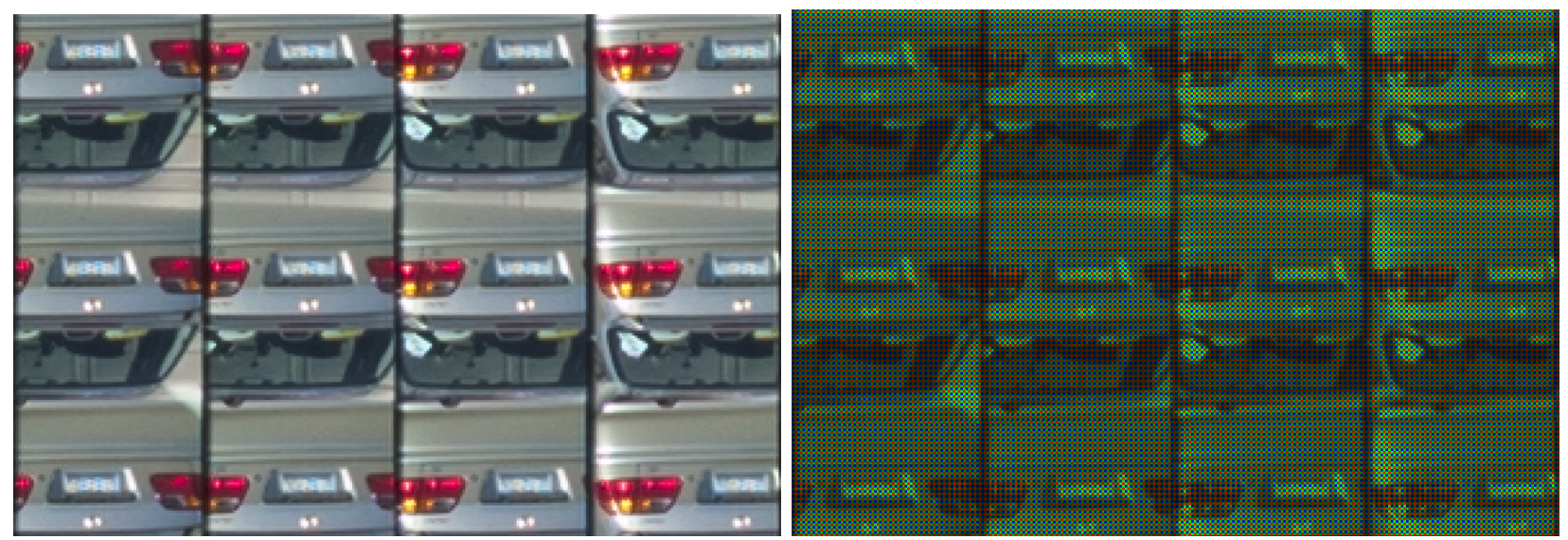

Figure 13. Left: Crop from a captured plenoptic image. Multiple copies of the license plate have been captured. The color image has been generated with conventional demosaicing. Right: Crop from a captured plenoptic image. Multiple copies of the license plate are visible. Pixel values and Bayer colors were extracted directly from the raw image.
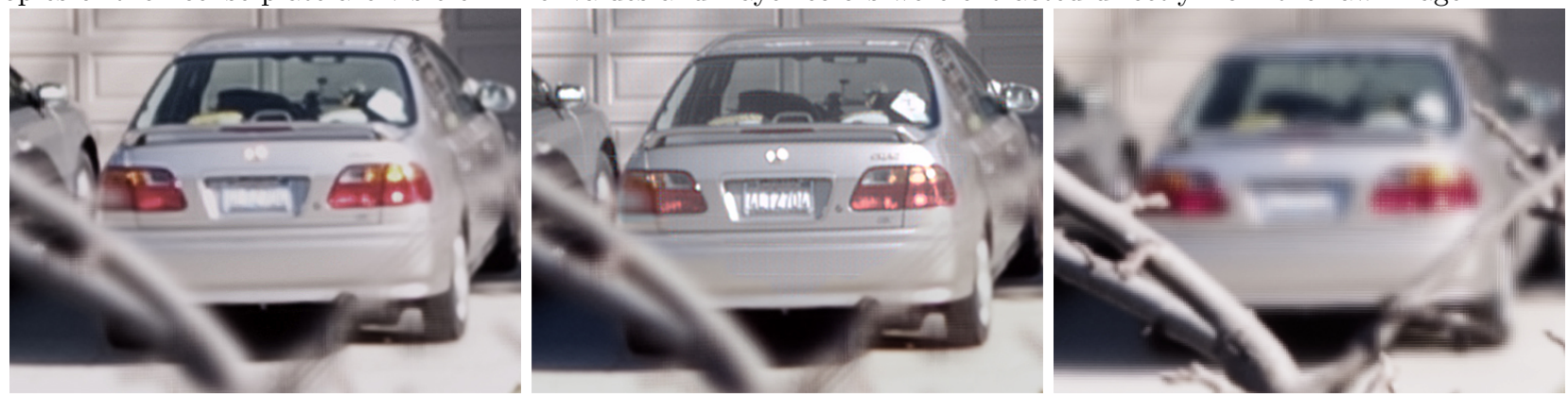

Figure 14. Superresolution with a Bayer filter array. Left: Superresolution with traditional Bayer demosaicing. Middle: Superresolution with our algorithm starting from raw data. The image is focused on the license plate. Right: Changing focus to the tree's branches.

\section{REFERENCES}

[1] Adelson, T. and Wang, J., "Single lens stereo with a plenoptic camera," IEEE Transactions on Pattern Analysis and Machine Intelligence, 99-106 (1992).

[2] Levoy, M. and Hanrahan, P., "Light field rendering," ACM Trans. Graph., 31-42 (1996).

[3] Lippmann, G., "Épreuves réversibles. photographies intégrales.", Académie des sciences , 446-451 (March 1908).

[4] Lippmann, G., "Épreuves réversibles donnant la sensation du relief," Journal of Physics 7(4), 821-825 (1908).

[5] Georgiev, T., Zheng, K., Curless, B., Salesin, D., and et al., "Spatio-angular resolution tradeoff in integral photography," Proc. Eurographics Symposium on Rendering (January 2006).

[6] Ng, R., Levoy, M., Brédif, M., Duval, G., Horowitz, M., and Hanrahan, P., "Light field photography with a hand-held plenoptic camera," Tech. Rep. CSTR 2005-02, Stanford University Computer Science (Apr. $2005)$.

[7] Ng, R., Digital light field photography, PhD thesis, Stanford University, Stanford, CA, USA (2006). Adviser: Patrick Hanrahan.

[8] Lumsdaine, A. and Georgiev, T., "The focused plenoptic camera," in [In Proc. IEEE ICCP], 1-8 (2009).

[9] Bishop, T. E., Zanetti, S., and Favaro, P., "Light field superresolution," in [International Conference on Computational Photography], (2009).

[10] Georgiev, T. G. and Lumsdaine, A., "Superresolution with plenoptic 2.0 cameras," in [Signal Recovery and Synthesis], Signal Recovery and Synthesis, STuA6, Optical Society of America (2009). 

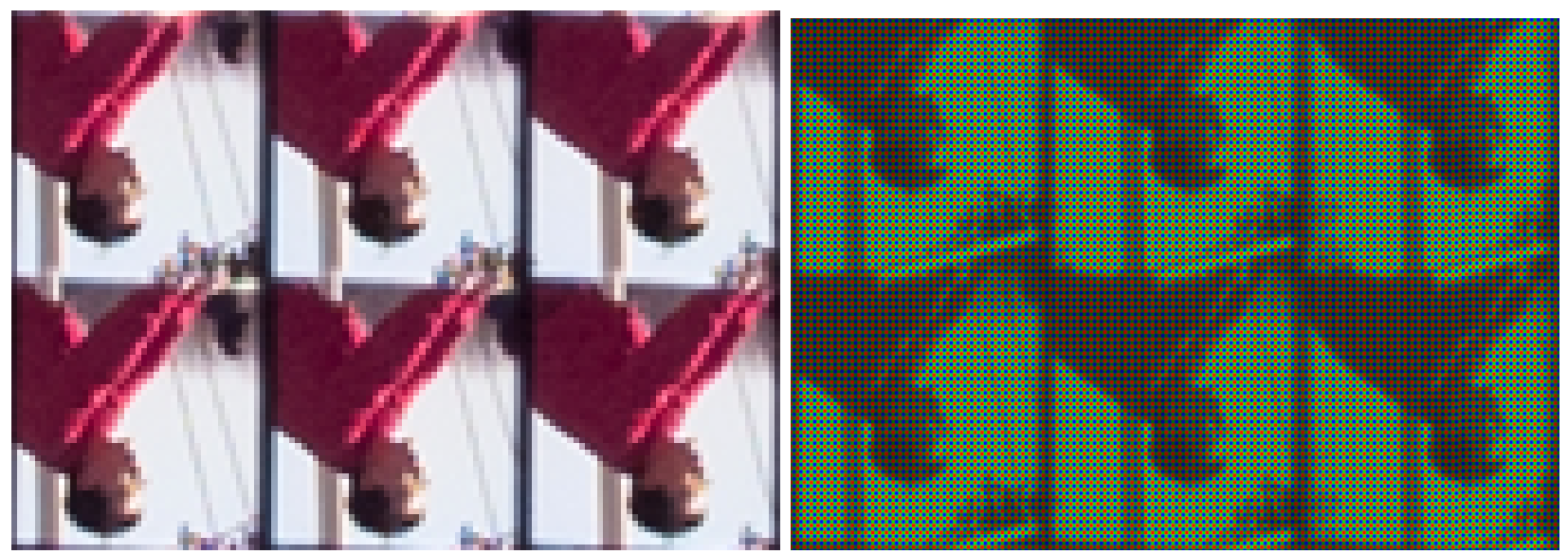

Figure 15. Left: Crop from a captured plenoptic image. Multiple copies of the person on the bike image have been captured. The image has been processed with conventional demosaicing. Right:Crop from a captured plenoptic image. Multiple copies of the person on the bike are visible. Pixel values and Bayer colors were extracted directly from the raw image.
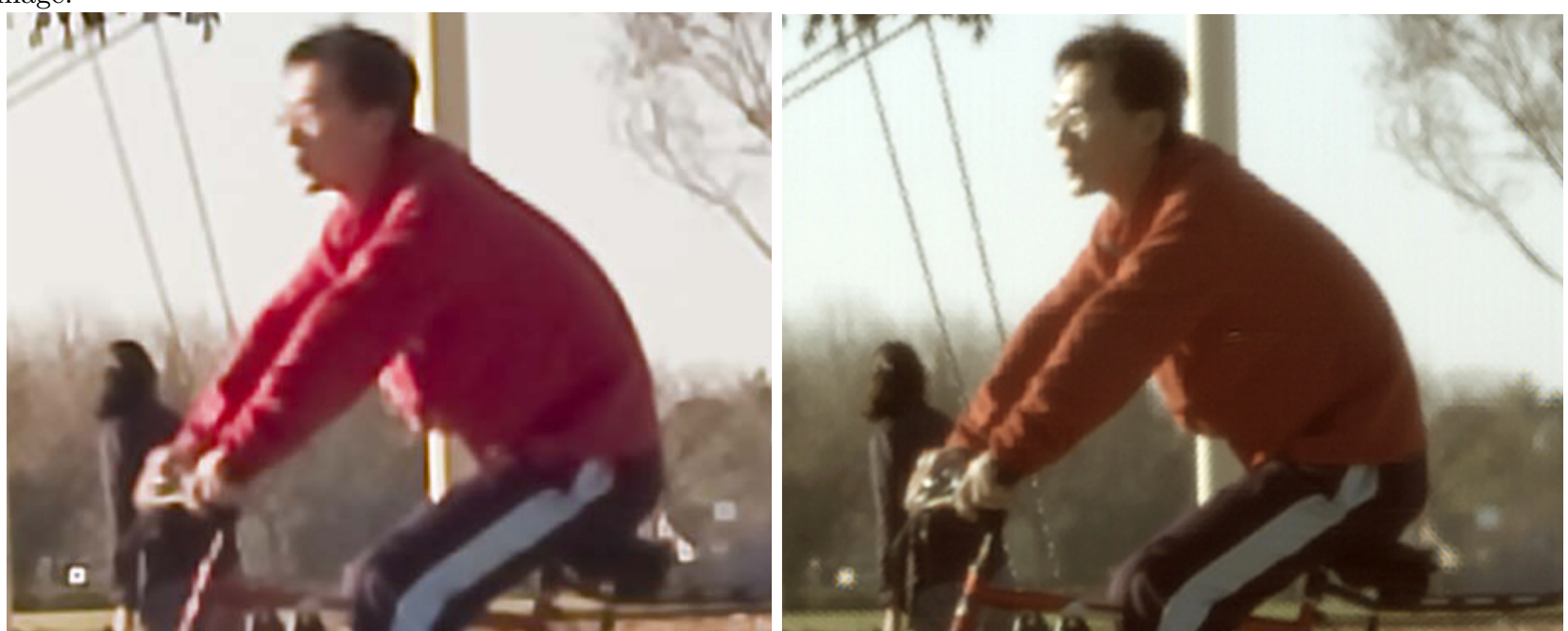

Figure 16. Superresolution with a Bayer filter array. Left: Superresolution with traditional demosaicing. Right: Superresolution from raw data with our algorithm. The image is focused on the bike. Notice that the swings hand on a chain, visible on the new superresolution algorithm (image on the right).

[11] Ng, M. K. and Bose, N. K., "Mathematical analysis of super-resolution methodology," Signal Processing Magazine, IEEE 20(3), 62-74 (2003).

[12] Park, S., Park, M., and Kang, M., "Super-resolution image reconstruction: a technical overview," Signal Processing Magazine (January 2003).

[13] Gotoh, T. and Okutomi, M., "Direct super-resolution and registration using raw cfa images," Computer Vision and Pattern Recognition, IEEE Computer Society Conference on 2, 600-607 (2004).

[14] Vandewalle, P., Krichane, K., Alleysson, D., and Ssstrunk, S., "Joint Demosaicing and Super-Resolution Imaging from a Set of Unregistered Aliased Images," in [Proc. ISET/SPIE Electronic Imaging: Digital Photography III], 6502 (2007).

[15] Zomet, A. and Peleg, S., "Multi-sensor super-resolution," Applications of Computer Vision, IEEE Workshop on $\mathbf{0}, 27$ (2002).

[16] Saito, T. and Komatsu, T., "Super-resolution sharpening-demosaicking with spatially adaptive totalvariation image regularization," in [Advances in Mulitmedia Information Processing - PCM 2005], Ho, 
Y.-S. and Kim, H., eds., Lecture Notes in Computer Science 3767, 246-256, Springer Berlin / Heidelberg (2005).

[17] Chai, J., Chan, S., Shum, H., and Tong, X., "Plenoptic sampling," ACM Trans. Graph., 307-318 (2000).

[18] Georgiev, T. and Lumsdaine, A., "Focused plenoptic camera and rendering," Journal of Electronic Imaging 19(2), 021106 (2010).

[19] Wolberg, G., [Digital Image Warping], IEEE Computer Society Press (1990). 